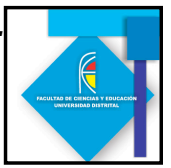

\title{
INCONVENIENTES CON LA TECNOLOGÍA EN LA ENSEÑANZA DE LA FÍSICA
}

\section{PROBLEMS WITH THE TECHNOLOGY IN THE TEACHING OF PHYSICS}

\author{
Juan Carlos Orjuela Abril ${ }^{1}$
}

\begin{abstract}
Resumen
El problema social en el que vivimos, nos muestra una serie de deficiencias en el ámbito de la educación de los jóvenes, bien sea en el hogar, en el colegio, en el trabajo, o en su entorno social (amigos). La drogadicción, el alcohol, el maltrato, la televisión, los video juegos, la moda, son condiciones sociales a las cuales los jóvenes están expuestos en su diario vivir, y por ende, estas influencias toman posesión en el proyecto de vida de los jóvenes, y no desarrollan su pensamiento en cosas que no ayudaran en su vida futura si no que por el contrario se podría estar perdiendo conocimiento en nuestra civilización, y es allí donde los docentes entran a actuar.
\end{abstract}

Palabras clave: Tecnología, enseñanza de la física, educación

\begin{abstract}
The social problem in which we live, shows us a number of shortcomings in the education of young people, whether at home, at school, at work or in their social environment (friends). Drug addiction, alcohol abuse, television, video games, fashion, social conditions are to which young people are exposed in their daily lives, and thus take possession of these influences in the life of young and do not carry out his thinking on things that do not help in their future lives if not on the contrary, knowledge can be lost in our civilization, and that is where teachers come to act.
\end{abstract}

Keywords: Technology, science of physics, education

\section{Introducción}

Una de las características fundamentales del papel del docente es formar a los jóvenes como personas "integras" (me refiero a integras por desarrollar un proyecto de vida), que

\footnotetext{
${ }^{1}$ Universidad Distrital Francisco José de Caldas - Semillero de astroingeniería, jcorjuelaa@correo.udistrital.edu.co
} 


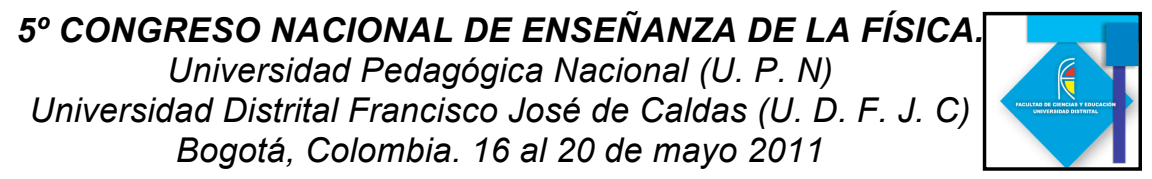

enfoquen su vida en su potencial característico, el docente es responsable del enfoque que tome cada una de las generaciones salientes.

Es cierto que la televisión, los amigos, la tecnología tienen un impacto muy fuerte en el desarrollo de una persona, pero hay que tener en cuenta, la manipulación de las mismas, ya que su enfoque esta dado al "consumismo caníbal", un enfoque donde no se respeta la integridad personal ni natural.

\section{La tecnología un arma de doble filo}

Hay que tener cuidado con el enfoque que le damos a la tecnología saliente, ya que puede servir para mejorar la calidad de vida de las personas, para ahorrar tiempo y todas esas bonitas presentaciones que nos dan, pero ¿Qué ocurre cuando no le damos un buen uso?. La tecnología se manifiesta como destructora del análisis, de las discusiones científicas en un aula de clase, de conocer la posición de los estudiantes.

El enfoque que debe dar el docente a la tecnología es en el ámbito de la discusión, de análisis, no se puede dejar una tarea que cause pereza mental si no que por el contrario, haga que los estudiantes se pregunten el por que de las cosas, de los fenómenos, hoy en día el Internet lo tiene todo y fue creado para facilitar, así como la calculadora y los sensores para ahorrar tiempo.

Teniendo en cuenta lo anterior, se ha visto que cuando le damos mal uso a estas herramientas, terminamos olvidando cosas importantes como lo es el análisis.

\section{Atacando de la misma forma}

Uno de los usos que podemos dar a estas tecnologías, es manipularlas de la misma manera que son utilizadas para captar la atención, y es allí donde los responsables de la educación debemos seguir avanzando, junto con la manera de ver de los estudiantes.

No puede ser una justificación el hecho de que por pertenecer a una generación mucho mayor nos reactualicemos, o porque los estudiantes no muestran interés "para que me esfuerzo", que el tiempo no alcanza para "dictar" los temas, el docente es el encargado de hacer que los estudiantes se interesen, y como el interés de cada generación es distinto por el entorno social, el docente debe ir de la mano con cada generación.

Tomare el ejemplo de un programa de televisión llamado el "profesor súper o", vemos como se utiliza la televisión para atacar la "ignorancia idiomática", allí enseñan cómo debemos utilizar ciertas palabras que son mal utilizadas a la hora de hablar, y es grato ver como la mayoría de los jóvenes tienen un grado de captación frente a este personaje. James Kakalios utilizó los comics para explicar ciertos fenómenos físicos a personas que no tenían un acercamiento con la física.

A mi parecer esta es la actitud que debe tomar el docente frente a la educación, es buscar el mejoramiento en conjunto con los estudiantes para que ellos y los docentes se enriquezcan en su diario vivir. 
Buscando al responsable

$5^{\circ}$ CONGRESO NACIONAL DE ENSEÑANZA DE LA FÍSICA.

Universidad Pedagógica Nacional (U. P. N)

Universidad Distrital Francisco José de Caldas (U. D. F. J. C)

Bogotá, Colombia. 16 al 20 de mayo 2011

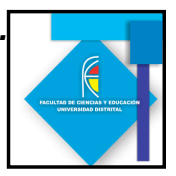

Teniendo en cuenta el papel que juega cada aspecto que conforma la sociedad, es difícil hallar un responsable de la catástrofe mental y por ende social en la que vivimos, pero lo que es cierto, es que si las personas que se han designado para la formación de los jóvenes falla, estas son las responsables en un gran porcentaje. No es posible que el docente le de pereza dar una clase, que no prepare un proyecto en el aula, que no se interese por razones políticas en la educación (evaluación, tiempo, etc.), se debe tener en cuenta que la razón fundamental por la cual se escogió ser docente es por enseñar, pero no enseñar como si fuera cualquier cosa, si no por el contrario de enseñarle a personas que tienen sueños, sentimientos, esperanzas y que hasta ahora comienzan en el camino de la vida, que están formando una personalidad y esta es una razón por la cual están abriendo puertas a las posibilidades que se les brinde, y si en el aula o colegio donde permanecen una gran mayoría de su tiempo no se les ofrece algo interesante, algo con lo cual se identifiquen, lo buscaran en algún lado (alcohol, drogas, televisión, moda, etc..), donde si llamaron su atención, donde si utilizaron la tecnología para atraerlos, pero donde no pensaron en formar.

\section{Referencias Bibliográficas}

http //:www:colciencias:gov:co=programaestrategia=cienciasbsicas;

http //:www:graduadoscolombia:edu:co=html=1732=article195063:html;

Didactica de las ciencias Experimentales;No36; pp; 547; abril2003; http //:www:portafolio:com:co=economia=economiahoy=ARTICULO WEBNOTAINTERIORPORTA6402447:html;

http //:www:mineducacion:gov:co=1621=propertyvalues41323tableropdf:pdf;

ESTAELLAB:R; (1995); Astronomiaenlaescuela; primeraedicion;Centrode publicacionesdelMEC; revisadoel15deseptiembrede2010

Division deformacion avanzadad el ICFES Bogota; mayo31de1983; http //:www:colombiaaprende:edu:co $=\mathrm{html}=$ estudiantesuperior $=1608=$ article198142:html; revisadoel15deseptiembrede2010

ALONSOM:ACOSTAV:(1972); Introduccion a lafisica; TomoI; pag858:

IGLESIASM;QUINTEROSC;GANGUIA; AstronomiaenlaEscuela :

Situacionactualyperspectivasfuturas;CEFIECFCEyNU

BAIntendenteGuiraldes2620;C1428EHA;CiudadAutonomadeBuenosAires:

http //:wwwgva:dec:usc:es=mjose=docencia=3ciclo=tema1:htm;

PORTILLAJ;ElementosdeAstronomadePosicin;Observatorioastronmico Nacional; FacultaddeCiencias; UniversidadNacionaldeColombia; mayo2001

KAKALIOS J. la física de los superhéroes 